GRAPE SUGAR AND GLUCOSE. By O. Luthy.

THE MANUFACTURE OF GLUCOSE AND GRAPE SUGAR. GRAPE sugar was first manufactured in France, in the
ear 1809, from grape juice. During the time of the torious continental blockade, ordered by Napoleon, the prices of sugar (of which only one kind, namely : colonial high, that cheap substitutes were eagerly looked out for. The grape juice was neutralized by powdered chalk, and
rapidly boiled down to a thin syrup of $20^{\circ} \mathrm{B}$. It was then cooled and filtered to free it from the lime salts it had deposited, and further evaporated to $32^{\circ}$ B., in which state, as stead of sugar. Boiled down to $45^{\circ}$ B., this syrup will, on cooling, deposit large quantities of granular grape sugar, gar, by draining in sugar monlds, or better by centrifugal machines: 1,000 parts of grapes yield 800 parts of must, ports of purape sugar.

About the same time, Kirchhoff, a chemist in St. Petersburg, found that starch by boiling with water and a little
sulphuric acid was changed into sugar. This discovcry created a great sensation, because it was believed that this starch sugar would replace cane sugar. It was soon experthe two, starch-sugar being less sweet and less soluble than colonial sugar, and its manufacture didn't become so important as was anticipated; so much the less, as in the same pe-
riod and by the same cause (the continental decree), the beet sugar industry got a great impulse and gradually ripened to its present success.
As a substitute, however, for Indian syrup (colonial moprocess, became favored and was manufactured in malt quantities (best molasses because of its bitter and salty taste the time, also, the solid starch sugar which was found to identical with grape sugar came into use, especially for the
improvement of wine-must in bad seasons. It then was em improvement of wine-must in bad seasons. It then was em-
ployed also in making fruit wines, and at last in manufacturing wine wholly artificial. In beer-brewing it is also Germany, 47 factories turned out over I1, 11,000 tons of starch or grape sugar, and nearly 15,000 tons of starch-syrup or
glucose (Wagner's Jahresbericht.) From other countries no statistics were obtained; no doubt, the amount would be
doubled, if France, Belgium and Holland were included in the report.

are manufactured mowadays from starch, is not quite so plain as it might first appear. When we add to a boiling hot thin starch-paste a small quantity (about two per cent. of the
starch) of sulphuric acid (oil of vitriol), previously diluted with water, it becomes at once liquid; the starch is rendered
soluble, changed into amiduline, as this new body is called, soluble, changed into amiduline, as this new body is called, By further boiling this is gradually converted in starch.
gum or dextrine, a substance which, when dry, resembles ways as a substitute for that substance. If the boiling cony tinues sufficiently long, the solution becomes sweet, the gum dextrine by degrees is turned into a new body again, and at
last, when the conversion is complete, we find nothing else in the solution besides a sweet body, which behaves exactly like grape-sugar, and the original amount of sulphuric acid
which has remained unchanged. On the time spent in boilWhich has remained unchanged. On the time spent in boil-
ing, and on the quantity of acid used, it depends whether the conversion is more or less complete; whether the starch case more or less gum dextrine, and even amiduline (soluble
starch merely) is left mixed with the sugar. If we apply
from one to two per cent. of acid only, and boil but for a from one to two per cent. of acid only, and boil but for a
few hours, the result is a product which is still rich in
gum dextrine, preventing, by its viscous properties, the ad. gum dextrine, preventing, by its viscous properties, the ad.
mixcd grape sugar from crystallizing, and which product mixcd grape sugar from crystallizing, and which product
consequently refuses to become solid. It is what is commer cially called glucose. If we, however, raise the amount of boil for eight or ten hours, the final result will be a fine granular solid grape sugar.
When the conversion of the starch into glucose or grape sugar is brought to the desired degree, we next have to ex-
tract and remove the acid. This is done by means of tract and remove the acid. This is done by means of
powdered chalk or slacked lime. The sulphuric acid com
bines with the lime, forming sulphate of bines with the lime, forming sulphate of lime, which de-
posits, leaving the solution sweet. After subsiding the posits, leaving the solution sweet. After subsiding the
liquor is filtered and the water boiled away, until glucose or sugar is left in the proper finished state. We see from
these outlines that the manufacture of glucose and grape sugar is divided in three principal processes, namely: con-
version, neutralization and evaporation. 1.

1. Conversion. - The boiling of the starch with the acid water is effected on a small scale in a copper kettle on a
naked fire, or in extensive factories in large wooden tubs by means of a large copper steam coil. The proper quantity of
water is first heated to boiling, then the requisite amount of sulphuric acid (oil of vitriol), previously mixed with three to five times its weight of water, is added; and whilst
the liquid is kept boiling continuously, the has been mixed with water to a milky consistency, is grad
ually poured in. The flow of the starch milk is so rated ually poured in. The flow of the starch milk is so regulated
that the liquor in the tub never cools below boiling, and never becomes cloggy or pasty.
To every 100 pounds of dry starch take 40 gallons of water in all, and 1 to 2 pounds sulphuric acid for glucose, added, the boiling is continued until the desired degree of conversion has taken place. Glucose will require about $2 \frac{1}{2}$
or 3 hours, grape sugar from 5 to 9 hours. From time to or 3 hours, grape sugar from 5 to 9 hours. From time to
time a sample is drawn to be tested. This is done at first by means of a solution of iodine (prepared by dissolving equal a coffee brown colored solution,) and afterwards by stron addition of a few drops of the iodine solution, or red on th converted into gum dextrine and grape sugar, and the boil If solid grape sugar is intended, the boiling must propared. til the alcohol test is satisfactory. A spoonful of the liquor is poured into a test tube, and shaken up with about five
times its bulk of strong alcohol. The gum dextrine being insoluble in alcohol will render the mixture turbid if pres ent, whilst grape sugar alone will remain dissolved on the During the germination of grains in the process of malt
addition of alcohol, the mixture thus being clear; therefore ing, a peculiar body (or mixture of bodies) is formed, named
the boiling is continued until all dextrine is completely con verted into grape sugar, when the alcohol test will not be
come milky any longer.
Instead of an open kettle or tub for boiling the acid starch
mixture a closed "converter" is used in many large facmixture, a closed "converter" is used in many large fac
tories. This is a strong cylindrical vessel of boiler iron lined with lead inside, and provided with a perforated
steam coil for heating, with pressure guage, thermometer, afety valve, etc. Water, acid and starch are introduced and heated as before; but after all the starch is in, the con-
verter is closed, and then by means of high-pressure steam bout 90 pounds. This temperature is kept up until conver-
bours on is complete. It is claimed for this process that the con version is done in less than half the time of the old way, and
that it is more entire, the resulting grape sugar being free of gum, and of a purer, finer taste.

2. Neutralization or Separation of the Sulph uric Acid from
the Sugar Solution. - When by a sufficiently long boiling the desired degree of conversion into either glucose or grape sugar is accomplished, the sulphuric acid must be removed
from the liquor. This end is reached by the addition of carbonate of lime (or technically, powdered chalk, marble or
limestone). This is decomposed by the acid, which commestone). This is decomposed by the acid, which com
bines with the lime and expels the carbonic acid gas, which causes the liquor to foam. Chalk or marble must be ground quantities (handfuls) only at a time, in order to prevent overflowing, the liquor being constantly stirred until the last
addition causes no more effervescence or bubbling. For
every pound of oil of vitriol applied, one pound of carbonevery pound of oil of vitriol applied, one pound of carbon-
ate of lime is required, but since chalk, marble or limestone ate of lime is required, but since chalk, marble or limestone
are not pure carbonate of lime, and an excess of them is of no harm, rather more, say $11 / 4$ pounds to every pound of
acid applied, may be taken. The chalk or marble powder is
best stirred up in water to a milk before it is poured into the best stirred up in water to a milk before it is poured into the As a neutralizing agent slaked lime has also been recommended and used. It has two advantages over chalk: it is
cheaper, and can easily be made into the finest milk. It
pplication is somewhat difficult, however, and dangerous to application is somewhat difficult, however, and dangerous to just enough is added, and the least excess of it will color
the sugar yellow or brown. Another quite serious trouble the sugar yellow or brown. Another quite serious trouble
with lime is caused by the large quantities of magnesia it
often contains-25 to 35 per cent. are quite usual in the different kinds of lime made and sold in the United States along
the Atlantic coast. Lime combines with sulphuric acid to the Atlantic coast. Lime combines with sulphuric acid to
make sulphate of lime, which is almost insoluble, and subsides nicely from the liquor on standing. Magnesia,
however, forms sulphate of magnesia, or Epsom salts, wowever, forms sulphate of magnesia, or Epsom salts, is very soluble, it does not deposit but remains in the liquor, After the neutralization of the liquor is effected, it
Aften rawn off from the converting vat or kettle into a tub or
ank below, and allowed to settle for 24 hours. This tank
hould be surrounded by a bad conductor, and well covered, hould be surrounded by a bad conductor, and well covered,
$\mathrm{n}$ order to prevent the cooling of the liquor, as fermentation will otherwise soon set in and turn it sour. When the sediment has pretty well subsided, the clear liquor is drawn off and boiled down (or evaporated, as chemists express them rering bags, which, after draining, are subjected

The remainder in the bags is good for manure. thin liquor has next to be boiled down to a syrup. This may
be accomplished in a shallow copper kettle over a low fire, or better by means of a steam jacket or coil. Modern deor better by means of a steam jacket or coil. Modern de-
mands as to color and flavor, however, are so great that a
vacuum pan can hardly be dispensed with. The evapora-
tion is carried on to about $2 \tilde{5}$ or $30^{\circ} \mathrm{B}=45$ or 55 saccharomen is carried on to about $2 \tilde{5}$ or $30^{\circ} \mathrm{B} .=45$ or 55 saccharo-
meter per cent., when the solution has acquired the consis-
tency of a thin syrup, and is now quite turbid again by the tency of a thin syrup, and is now quite turbid again by the
separation of sulphate of lime, which had been dissolved.
To get rid of this the syrup is drawn off again into subsiding tubs, and allowed to settle for a few days, or it is filtered ness and color,bone charcoal filtersare provided. 'They consis in cylindrical tanks, rather high, with sieve bott
with a cloth and filled with granular bone-black.

In large establishments these filters are of sheet or cast
iron, from 2 to 6 feet wide and more, and from 10 to 30 feet high. For small manufacture wooden ones, such as old whis is removed, and the other provided with a number of small holes to serve as false bottom. It is covered with a suitable used in sugar refining in general. The thin liquor is poured
upon, and, slowly and evenly passing through the animal upon, and, slowly and evenly passing through the animal
charcoal, leaves all color and mud behind, and runs off white and clear, the bone charcoal absorbing impurities and
coloring-matter. When its power is exhausted, it is washed coloring-matter. When its power is exhausted, it is washed
with boiling hot water, dried and reburnt, and may then be The clear decolored thin syrup is drawn back into the
The vacuum pan and boiled down. If the conversion was done with a view of making starch-syrup or glucose, the boilin
is continued till the syrup gauges hot $40^{\circ} \mathrm{B}$, when the product is ready for the market. On cooling it will form
a very heavy $\left(45^{\circ}\right)$ thick syrup, clear and colorless, or almost a very heavy $\left(45^{\circ}\right)$ thick syrup, clear and colorless, or almost
so, of an agreeable pure taste, sweet and mucilaginous at shouldn't turn sour or mouldy.
should not deposit

In the other case, if grape sugar is intended, the boiling is
shouln't In the other case, if grape sugar is intended, the boiling is
discontinued when the contents of the pan gauge $38^{\circ}$ or
$40^{\circ}$. They are then also drawn off in crystallizing tanks, and allowed to cool and congeal. The resulting crystalline
mass is freed from syrup by strong pressure, after which it mass is freed from syrup by strong pressure, after which it
is remelted and cast into moulds; or, if a less pure article is
desired, the syrup is evaporated in the pan (to about $44^{\circ}$ ) till desired, the syrup is evaporated in the pan (to about $44^{\circ}$ ) till
on cooling it hardens completely. It is run into packing on cooling it hardens completely. It is run into packin
boxes directly, and, while cooling, stirred occasionally to promote the formation of a uniform grain.

The manufacture of grape sugar from pulp (the waste of spoken of, has never acquired any practical shape. Not pound and more to every pound of rags), but the resulting product is so impure that it could never be use or sweetening purposes. It might be put to fermentation cheaper raw materials for the same product.

MANUFACTURE OF GLUCOSE BY MEANS OF MALT.

During the germination of grains in the process of malt-
ing, a peculiar body (or mixture of bodies) is formed, named
diastase, which has the mysterious power (simular to sul- phuric acid) to turn starch into grape sugar and dextrine
gum, at a temperature of $140^{\circ}$ to $165^{\circ}$. Such grains, which gum, at a temperature of $140^{\circ}$ to $165^{\circ}$. Such grains, which
have been made to germinate by soaking in water and spreading in thin layers on floors for from six to fourteen days, and by the name of malt, and used extensively in brewing and distilling, for the conversion of the starch into sugar in the
process of mashing. process of mashing.
For the conversion of every 100 parts of starch, from 5 to 10 parts bruised malt are required, with 350 to 400 water.
Water and malt are heated to $140^{\circ}$ to $155^{\circ} \mathrm{F}$; t then the starch added, previously stirred up with water, and with constant stirring the temperature is raised to $160^{\circ}$ and $165^{\circ}$ sugar. When the conversion is complete the heat is raised dowz: - The resulting syrup is very gummy, light-brown
colored, and has a peculiar taste, by no means disagreeable, colored, and has a peculiar taste, by no means disagreeable,
resembling that of malt. However, it is subject to souring and moulding, and very much so; when, instead of starch

It remains to say something of the different uses of glucose It remains to say something of the different uses of glucose
and grape sugar. The largest quantity of grape sugar is probably consumed for the purpose of improving wines. This is done on the following principles :

When the proportion between the chief constituents of
the must, sugar, acid, and water are such that no good wine would result on fermentation, these proportions are altered and improved by the addition of grape sugar. When in a bad season the must is poorer in sugar but richer in acid
than in a good one, by the addition of the proper quantity of grape sugar and water the equilibrium is restored.
In countries wherein the grape juice acid is always in exIn countries wherein the grape juice acid is always in ex-
cess, certainly this method of improvement (called after its
inventor, Gall's method), is generally taken advantage of. Another way of using grape sugar in preparing wine was taught by Petiot. The husks of the grapes, after expressing the juice, still contains enough extractive matter (acids, Gether with a solution of sugar of proper strength.

rom fruits, as currant, gooseberry, etc., wine, the acid predominating always in these fruits. Also in the manu-
facture of artificial wine (of which a very large quantity is consumed in this country) grape sugar plays an important part. In beer-brcwing also an enormous quantity of this
sugar is worked up. We state on the authority of Prof. sugar is worked up. We state on the authority of Prof.
Wagner, in Wurzburg, Bavaria, that to every three cwt. of malt one cwt. of potato-sugar is empioyed. A large quancoloring," originally used for coloring cordials, etc., then to imitate rum or cognac, and to give whiskey its proper gone far enough, also lager beer receives its dark color by burnt sugar. That glucose and grape sugar are also largely need to mention here.
As with commercial cane sugars, so also with glucose and grape sugar; there is a great difference in quality, and no easy way of establishing their real value. Pure taste, absence of color, perfect transparency, and great density are
the points by which glucose may be judged. Pure grape
sugar should be perfectly white, opaque, of a dry large grain, and, like glucose, have a pleasant taste.
There seems to prevail quite a popular prejudgment that There seems to prevail quite a popular prejudgment that
neither strong glucose nor hard grape sugar can be made corn, containing an oil, produces a disagreeable flavor. A more unfounded superstition than this is hardly possible, sugars" of different European manufacturers as there is between "potato sugar" on one hand and "corn sugar" on
the other; and as to the corn oil, I am of the opinion that it is pretty well removed during the preparation of the
starch. The potato sugar manufacturer has quite a similar starch. The potato sugar manufacturer has quite a similar (as Otto reports) a starch-sugar factory in Braunschweig,
Germany, had to be removed out of town as a public nuisBy looking to a complete conversion, a most careful sepaBy looking to a complete conversion, a most careful sepa-
ration of the acid, and a proper use of bone charcoal, the diffcome.

CHRYSOLIN, A NEW YELLOW DYE DERIVED By F. Reverdis.

THE coloring matter of which we are about to speak, and which we have prepared since March this year at the works of MM.P. Monnet $\& \mathrm{Co}_{\text {, }}$ at Geneva, is formed by the simul-
taneous action of phthalic and sulphuric acid upon benzylresorcin. he chloride of benzyl to act upon resorcin in presence of a small quantity of zinc.powder, or by heating an alkaline or, lastly, by heating to about $150^{\circ}$ in the oil-bath, a mixture
of 1 molecule of resorcin and of 2 molecules of chloride of benzyl.

The most simple manner of preparation consists in adding
he chloride of benzyl, little by little to the melted resorcin a large quantity of hydrochloric acid escapes, and the mass
becomes a reddish-brown. When all the chloride of benzyl has been introduced it is heated to $150^{\circ}$ in the oil-bath in a
flask fitted with an ascending c ondenser until the escape of lask fitted with an ascending condenser until the escape of
hydrochloric acid is at an end. The product of the reaction hydrochloric acid is at an end. The product of the reaction
s poured into water, boiled to expel the last traces of chlo ide of benzyl, let settle, and decanted.

The compound thus obtained is a strongly-colored oil,
com in a very elevated temperature, with partial decomposition. It decided green fluorescence. Benzyl-resorcin is soluble also
dissolo Preparation of Chrysolin.-The following method dispenses with the previous preparation of benzyl-resorcin: We
heat in the oil-bath to $130^{\circ}$ to $140^{\circ}$ in a retort of enamelled

Sulphuric acid

460 grms.
1 kilo.

The letter substance is transformed, in this operation, into

phthalic anhydride. We then introduced into the retort:

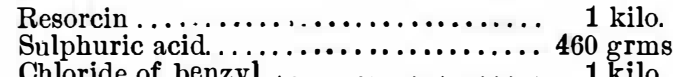


and heat gently in the water-bath. The heat may be re-
moved when hydrochloric acid begins to escape, and the reaction continues spontaneously. When no more hydrochloric acid is evolved, which may ensue in three or
four hours, the reaction is completed by heating for 12 solid product of the reaction solid product of the reaction is broken up and dissolved in
dilute caustic soàa. It is well to boil for a considerable time. When the residue no longer diminishes in volume, of hydrochloric acid. The precipitate is washed with cold water, dissolved in the quantity of carbonate of soda needful
to saturate the acid, and evaporated to dryness. The sodato saturate the acid, and evaporated to dryness. The soda-
salt of benzylated fiuorescein constitutes chrysolin. Chrysolin appears, as a mass, with green metallic refiec-
tions, but is red-brown when reduced to powder tions, but is red-brown when reduced to powder. It is solu. magnificent green fiuorescence, are precipitated by acids in magnificent gocen fiuorescence It yields bromated, iodated, and nitro-
derivatives, which are all beautiful coloring matters. Chrysolin may be fixed directly upon silk and wool.
Wool, however, is preferably mordanted in a beck of acetate of lead and alum. Cotton is mordanted with sulphate of alumina and dyed at a hand-heat. The shade of chrysolin approaches that of turmeric, and it
resists the action of light well.

Yellow coloring matters may also be obtained by replacing the chloride of anzyles of the fatty series. Methyl-resorcin, prepared by
heating under pressure the resorcinate of soda in alcoholic heating under pressure the resorcinate of soda in alcoholic
solution with chloride of methyl, yields also a yellow coloring matter.-Moniteur Scientifique.

\section{LABORATORY NOTES.}

By T. A. Edison.

HARD rubber or vulcanite, placed for several weeks in ni
trobenzol, becomes soft and pliable-like leather, and easily broken.
2. The vapor of chloral hydrate is a solvent of cellulose. I have found the corks of bottles containing the crystals being resolved into a black semi-liquid. Certain kinds of tissue paper are partially dissol
bottle containing the crystals.

3. A very difficult substance to dissolve is gum copal. I have found that aniline oil dissolves it with great facility. 4. Hyposulphite of soda is apparently soluble to a consid-
erable extent in spirits of turpentine. Targe crystals of "hypo" melt down to a liquid after several weeks, and if
the bottle be shaken, partially disappear. The turpentine smell nearly disappears. 5 . The vapors of iodine, in the course of
will penetrate deeply into lumps of beeswax.

6. If to a solution of bisulphide of carbon there be added twice its bulk of potassic hydrate in sticks, and the bottle be well sealed, the whole will, in two months, become an in-
tense reddish, syrupy liquid, with scarcely any free bisulphide of carbon.

on the sides of the bottles containing them, generally above on the sides of the bottles containing them, generally above
the water line. Among such solutions in $100 \mathrm{c}$. c. of rain water may be mentioned a 14 -grm. solution of a cetate of
uranium, 8-grm. do. of proto-acetate of copper. 5 - 9 rm acetate of morphine, 10-grm. do. of formate of copper, 20 -grm. do. of tannate of iron. These deposits invariably
take place on that part of the bottle most exposed to light. take place on that part of the bottle most exposed to light.
This phenomena may be due to heat, but deposits or films occur in some solutions wittin the liquid as well as above it -especially noticealle with tannate of iron, the fil
which adheres strongly to glass. - American Chemist.

NEW METHOD OF DETERMINING PHOSPHORUS, ARSENIC, SULPHUR, CHLORINE, BROMINE AND By M. G. Brugelmans.

THe method proposed by the author consists in burning the organic matter in a current of oxygen and in conor of soda lime in the case of the determination of bromine
and iodine. We operate in a tube of Bohemian glass open and iodine. We operate in a tube of Bohemian glass open at both ends, of an internal diameter of $12 \mathrm{~m}$. m. Here are
introduced successively - First, a leaf of platinum of the width of 2 centimeters, rolled in a spiral, and forming a plug. heaped lightly so that it may occupy all the diameter of the is quite sufficient to retain completely the elements to be determined. Third, a second bed of platinum of a width of 6
centimeters rolled into a spiral; when the body in question centimeters rolled into a spiral; when the body in question contains phosphorus or arsenic, this spiral should be replaced
by a layer of fragments of very small infusible glass. Fourth, by a layer of fragments of very small infusible glass. Fourth, (at ture slightly elevated combustible gases or vapors. Fifth, the substance introduced directly into the tube, or contained in a small boat or vial, may be in large pieces. Finally, the tube is closed by the stopper holding a very narrow tube
(diameter $0.5 \mathrm{~m} . \mathrm{m}$.), through which the oxygen is transmitted; the extremity of the tube remains open. The total mence by heating the first half of the layer of lime, then oxygen is caused to enter, whilst the other half is raised to redness; at this moment we commence the combustion of the substance. The oxygen ought always to be in excess in the tube, so that the products arriving on the lime are entirely
burnt; in any case the lime should not blacken. The speed of the current of oxygen is liable to variations, but on the
average 100 c.c. may enter per minute. If we burn volatile matters or substances which are de-
If composed at a low temperature, giving off vapors, we cannot always prevent explosions, even on heating with great pre-
caution; in this case we commence the combustion in a current of air, and only turn on oxygen at the moment when we
have driven by heat all the substance into the layer of asbestos. Substances richin phosphorus are mixed with three times their weight of lime and placed in a large platinum boat. When
the combustion is finished we break the tube at the place the combustion is ninished we break the tube at the place the outside of the tube, and we heat the contents with water to clean it completely; then we add gradually nitric acid until the whole is in solution; in this way we only employ a
slight excess of nitric acid. It is well understood that the slight excess of nitric acid. It is well understood that the
tube and boat ought to be rinsed carefully in weak nitric \begin{tabular}{l|ll} 
acid. In this solution the haloid elements are precipitated & should be submitted to slight pressure. It intensifies its ef \\
in the state of chloride, bromide, or iodide of silver, where & fect, but very good results have been obtained when the
\end{tabular} $\begin{array}{lll}\text { they are determined volumetrically by Volhard's process by } & \text { fect, but very good results have been obtain } \\ \text { emulsion has simply been drained and dried }\end{array}$

me of two standard solutions of sulphocyanide of amb- In order to render the desiccation more rapid, and at the nium and nitrate of silver, ferric sulphate serving as an indi- same time more complete, I dry the precipitate in blotting cator. Let us add, however, that a part of the iodine is paper, and introduce it under a bell-glass, together with a
found in the state of free iodine and of iodic acid, and ought
dish containing some concentrated sulphuric acid, and make be brought back previously to the state of hydriodic acio orized.

Sulphuric acid is determined as sulphate of barium, orelse volumetrically by a standard solution of chloride of barium; after the method of Wildenstein, which the author has mod-
ified slightly. Finally, the phosphoric or arsenic acids are volumetrically determined with nitrate of uranium. The diameter exceeds 1 millimeter; they ought to be free from chlorine, sulphur, phosphorus, iron and aluminum. The author describes the preparation of pure lime and soda-lime;
for this latter he employs 4 parts of pure lime and 1 part of pure soda prepared from sodium.

The method seems to us to present a great interest for determining chlorine, sulphur or phosphorus in substances
which are poor in these elements, such as vegetable and aniwhich are poor in these elements, such as vegetable and ani-
mal products; it enables us, in fact, to burnín a short time a large quantity of matter (about 20 grms. in two hours).-

ALUMINIUM NiTRIDE.-Mallet hasmade some experiments bles iron in being ascertaining whether aluminium resem heated to very high temperatures with carbonate of soda, a particles, as well as crusts of yellow color, were observed on the surface or in the cavities of the aluminium regulus, an these proved to be a nitride of that metal. When amorphous the substance is pale yellow, when crystalline it is honey-
yellow and transparent. The crystals are lustrous, brittle, by water, either hot or cold, but by long exposure to moist air they become sulphur-colored and opaque, and finally crumble to a white powder (alumina), ammonia being given off. They are decomposed by acids and caustic alkalies
The composition of the pure substance was found to be;

Aluminium.

$66 \cdot 16$
$33 \cdot 84$

$100 \cdot 00$

which corresponds with the formula $\mathrm{Al}_{2} \mathrm{~N}_{2} .-A n n$. der Chem

A New Carbo-Hrdrate in Mrlk.-Ritthausen has found in milk a carbo-hydrate which is not milk sugar. It occurs only in small quantities, and is easily soluble in water; its
solution forms with a small quantity of the copper solutio and potash the well known blue liquid; when boiled for a
long time, a little cuprous oxide is deposited; if the liquid be previously boiled with a little sulphuric acid the application of a gentle heat is sufficient to cause a copious reduction.
Alcohol throws down a fiocculent precipitate from an aqueus solution of the substance. When evaporated to dryness, over sulphuric acid, its solution leaves a gum-like viscous residue, in which the granular, non-crystalline substance can consideration is not milk sugar.

\section{ON MIXING EMULSIONS}

I Have for some time past been occupied in experimentin on a mcans whereby emulsions can be made in large quant

nitrate of silver.
The agitation of the emulsion does not present any diffcultywhen made in smallquantities; but when manufactured on a large scale it is inconvenient to obtain the necessary
agitation. In order to remedy this I have had recourse to a

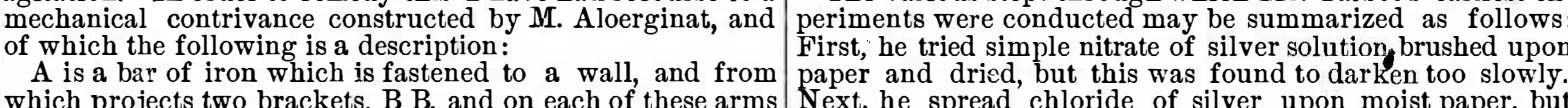
\begin{tabular}{l|l} 
of which the following is a description: & periments were conducted may be summarized as follows: \\
A is a bar of iron which is fastened to a wall, and from & $\begin{array}{l}\text { First, he tried simple nitrate of silver solution, brushed upon } \\
\text { paper and dried, but this was found to darken too slowly. }\end{array}$ \\
which projects two brackets, B B, and on each of these arms & Next, he spread chloride of silver upon moist paper, but
\end{tabular} are rings, C C C C. From these rings are suspended four this also proved unsatisfactory. He then discovered that

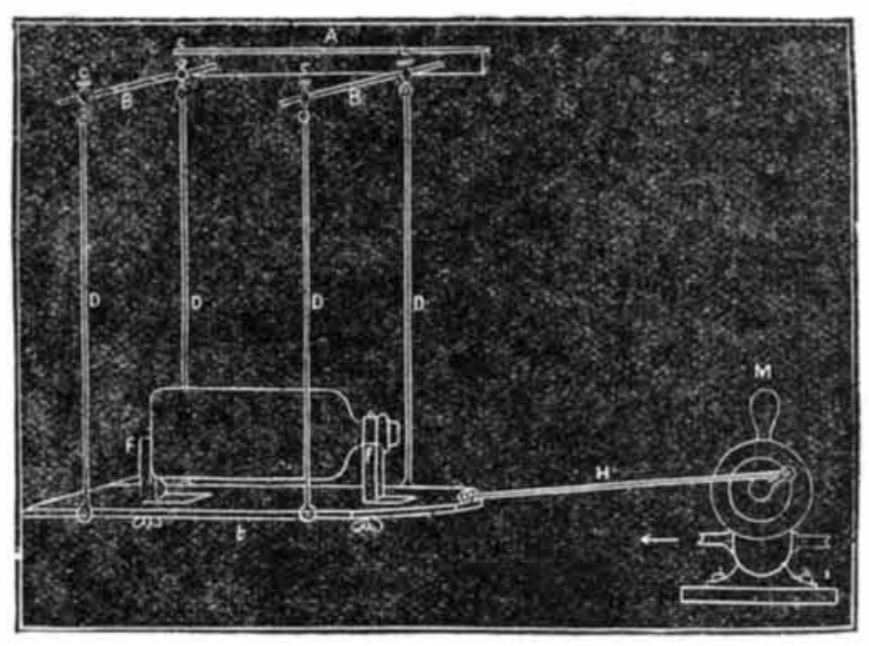

rods, D, terminated at each end by a ring or hook. These the best way by which to prepare chloride of silver was by rods support the platform, E, on which the bottle containing
the solution is placed. F F are supports for said bottle. The decomposition, the paper being first impregnated
with common salt and afterward with nitrate of silver. He

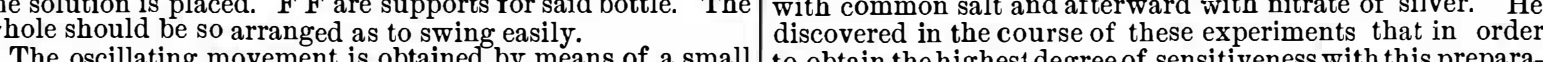

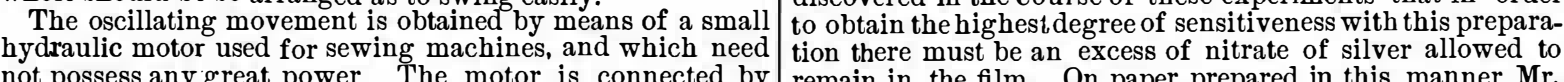

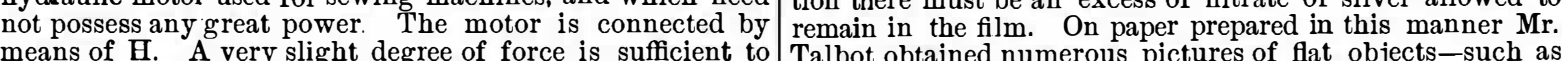
means of $\mathrm{H}$. A A very slight degree of force is sufficient to
oscillate a great quantity of emulsion when once it has been oscillate a great quantity of emulsion when once it has been
leaves, lace, and antlogous objects-by superimposing them
upon the sensitive paper and then exposing them to light set in motion. I have under these conditions made a large
quantity of emulsion, the results of which have been highly
But by means of a modification of this process-that is, by qatisfactory.

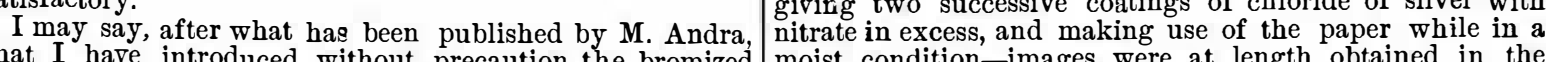
collodion in the hot alcoholic solution of nitrate of silver,
camera obscura, although a long exposure had to be given. without troubling myself about the precipitate.
The precipitation of emulsion with hot water is very easy;
but I think it ought to be remarked that the precipitate
periont will be found in the Philosophical Magazine at that
period. 